${ }^{1}$ Кафедра хирургической стоматологии Института стоматологии им. Е.В. Боровского ФГАОУ ВО «Первый МГМУ им. И.М. Сеченова» (Сеченовский Университет), Москва, Россия

${ }^{2}$ Кафедра эндокринологии №1 Института клинической медицины им. Н.В. Склифосовского ФГАОУ ВО «Первый МГМУ им. И.М. Сеченова» (Сеченовский Университет), Москва, Россия

Витамин D принадлежит к группе жирорастворимых секостероидов, играющих важную роль в организме человека. Его основными природными формами являются витамин $\mathrm{D}_{3}$ (холекальциферол) и витамин $\mathrm{D}_{2}$ (эргокальциферол). Их наиболее изученные функции - регуляция фосфорно-кальциевого обмена и обеспечение адекватного ремоделирования костной ткани. Исследования последних лет выявили также и «внекостные» эффекты витамина D, что позволило убедиться в более широкой роли данного соединения. Участие активных форм витамина D в процессах иммуномодуляции, оказание противовоспалительного, антимикробного, антипролиферативного эффектов и стимуляция клеточной дифференцировки отражаются и на заболеваниях челюстно-лицевой области.

В этой статье мы рассмотрели основные функции витамина D в организме человека, «внекостные» эффекты, механизмы его действия и влияние данных процессов на возникновение и течение заболеваний полости рта. Выявление взаимосвязи дефицита витамина D и процессов остеоинтеграции, костного ремоделирования, тяжести течения хронического рецидивирующего афтозного стоматита, плоскоклеточной карциномы и пародонтита позволило сделать вывод о целесообразности своевременной диагностики дефицита витамина D и коррекции его уровня в сыворотке крови у стоматологических больных.

КЛЮЧЕВЫЕ СЛОВА: дефицит витамина D; остеоинтеграция; дентальная имплантация; пародонтит; слизистая полости рта.

\title{
VITAMIN D IMBALANCE EFFECTS ON DENTOALVEOLAR SYSTEM: A REVIEW
}

\section{(c) Ekaterina Yu. Diachkova1*, Daria O. Trifonova', Maria O. Ibadulaeva², Guzel E. Runova², Valentin V. Fadeyev²,} Svetlana V. Tarasenko'

'Department of Dental Surgery of Borovskiy Institute of Dentistry

Federal State Autonomous Educational Institution of Higher Education I.M. Sechenov First Moscow State Medical University of the Ministry of Health of the Russian Federation (Sechenov University), Moscow, Russia

${ }^{2}$ Department of Endocrinology №1 of the Sklifosovsky Institute of Clinical Medicine

Federal State Autonomous Educational Institution of Higher Education I.M. Sechenov First Moscow State Medical University of the Ministry of Health of the Russian Federation (Sechenov University), Moscow, Russia

Vitamin D is a fat-soluble secosteroid that plays an important role in the human body. There are two main native forms vitamin D3 (cholecalciferol) and vitamin D2 (ergocalciferol). The regulation of calcium-phosphate metabolism and ensuring adequate bone remodeling are the most studied function of vitamin D. In recent years, researchers have found out the «extra-bone» effects of vitamin D and it allows us to be convinced of the great role of this compound. The participation of active forms of vitamin $D$ in the processes of immunomodulation, anti-inflammatory, antimicrobial, antiproliferative effects and stimulation of cell differentiation are reflected in diseases of the maxillofacial region.

In this article, we examined the main functions of vitamin D in the human body, the mechanisms of its action and influence on the occurrence and course of oral diseases. The discovery of the relationship between vitamin D deficiency and the processes of osseointegration, bone remodeling, the severity of chronic recurrent aphthous stomatitis, squamous cell carcinoma and periodontitis allow us to conclude that it is advisable to diagnose vitamin D deficiency in appropriate time and correction its serum level in dental patients.

KEYWORDS: vitamin D deficiency; osseointegration; dental implantation; periodontitis; oral mucosa.

Витамин D относится к группе жирорастворимых секостероидных соединений. Его поступление в организм обеспечивается двумя путями: алиментарным (с принимаемой пищей и пищевыми добавками) и посредством эндогенного синтеза в коже под действием ультрафиолетового излучения.
Широко распространенная недостаточность витамина D во всем мире повысила интерес к данному соединению и потребовала более детального изучения его влияния на различные органы и системы человека [1, 2].

Наиболее изученные и доказанные эффекты витамина и его производных - регуляция фосфорно-кальциевого 
обмена и костного ремоделирования. Связываясь с внутриклеточным рецептором клетки-мишени, витамин D инициирует продукцию генов, увеличивающих синтез белков, которые обеспечивают всасывание кальция и фосфора в кишечнике, транспорт ионов кальция из энтероцитов в кровь, что обеспечивает поддержание уровня кальция, необходимого для минерализации костной ткани. В почках витамин D увеличивает реабсорбцию кальция и фосфора. Также витамин D стимулирует костную резорбцию посредством активации остеокластов через активацию остеобластов.

Кроме того, обнаружение рецепторов витамина D во многих клетках и органах, например, макрофагах, моноцитах, дендритных клетках, клетках плаценты, паращитовидной железы и простаты, остеобластах, клетках гладкой мускулатуры и эпителиоцитах десневого прикрепления, способствовало открытию его «внекостных» эффектов [3, 4]. Участию витамина D отводят значительную роль в развитии иммунных процессов, в формировании противовоспалительного и антимикробного компонента, в замедлении клеточной пролиферации и стимуляции дифференцировки клеток.

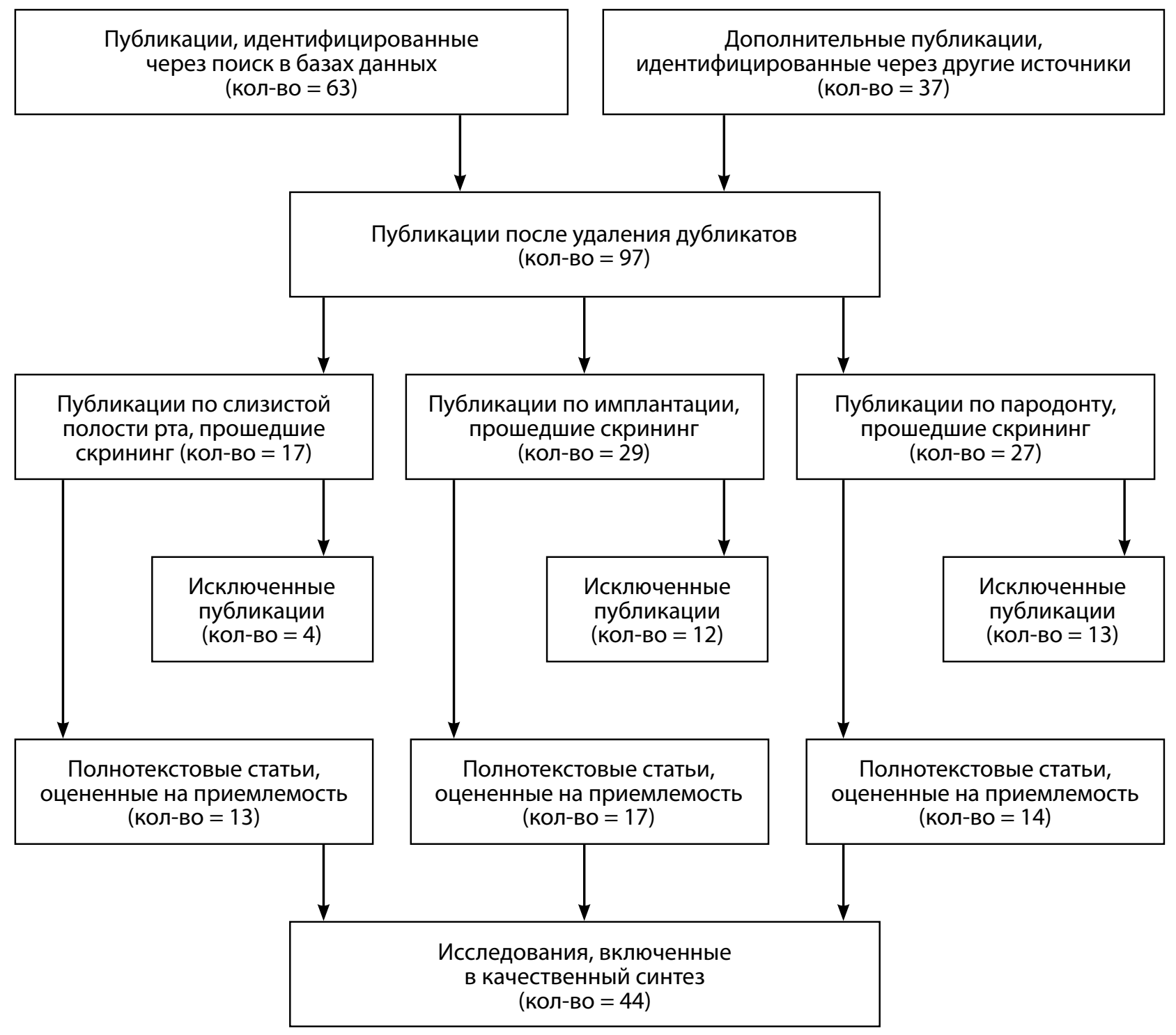

Рисунок 1. Схема отбора статей для систематического обзора.
Вопрос влияния витамина D на челюстно-лицевую область в современной стоматологии остается малоизученным и требует проведения дальнейшей исследовательской работы.

В настоящей статье представлен обзор современной литературы о влиянии витамина D на твердые и мягкие ткани ротовой области.

\section{МАТЕРИАЛЫ И МЕТОДЫ}

Основной поиск осуществлялся в базе данных PubMed (www.ncbi.nlm.nih.gov/pubmed), Google Академия (https://scholar.google.ru/schhp?hl=ru), elibrary (https://www.elibrary.ru) с использованием комбинации ключевых слов: "дефицит витамина D", “остеоинтеграция", "пародонтит", “дентальная имплантация", "слизистая полости рта" "vitamin D deficiency", "osseointegration", "dental implantation", "oral mucosa", "periodontitis". Временные критерии поиска - 2011-2020 гг. Соответствующая дополнительная литература была включена после ручного поиска в списках литературы включенных статей. Журналы, посвященные эндокринологии, имплантации и пародонтологии, были изучены вручную, с целью поиска статей (рис. 1).

\section{через иден (кол-во $=63$ )}




\section{МЕТАБОЛИЗМ ВИТАМИНА D}

Существует две основные природные формы витамина D: витамин $\mathrm{D}_{2}$ (эргокальциферол), который содержится в растительной пище (дрожжах, грибах, зерновых культурах) и поступает в организм только с продуктами питания, и витамин $\mathrm{D}_{3}$ (холекальциферол) - животного происхождения, который содержится в рыбе, сливочном масле, молоке, яйцах и может поступать в организм как с пищей, так и путем синтеза в коже из провитамина $\mathrm{D}_{3}$ (7-дегидрохолестерол) под действием УФ-лучей. Производные витамина D попадают в межклеточное пространство, а затем, связываясь с белками крови, в кровеносное русло. Обе формы являются неактивными и подвергаются дальнейшему метаболизму в организме. Первоначальное гидроксилирование происходит в печени под действием 25-гидроксилазы до 25(OH)D (кальцидиола). 25(OH)D является основной циркулирующей формой витамина D. Именно этот показатель используется для количественной оценки сывороточного уровня витамина D в клинической практике, так как имеет длительный период полувыведения - до 3 нед [5]. Согласно рекомендациям Российской ассоциации эндокринологов, адекватным уровнем витамина D считается показатель более 30 нг/мл (75 нмоль/л). Рекомендуемые целевые значения 25(OH)D при коррекции дефицита витамина D - 30-60 нг/мл (75-150 нмоль/л), снижение до 20 нг/мл (50 нмоль/л) рассматривается как недостаточность, а показатели ниже 20 нг/мл интерпретируются уже как дефицит витамина D [2].

Последующий этап метаболизма витамина D, катализируемый 1а-гидроксилазой, происходит главным образом в почках, в меньшей степени - в костной ткани, легких, печени, околоушных железах и кератиноцитах с образованием 1,25-дигидроксивитамина D (1,25(OH) 2 р или кальцитриола), который является конечной активной формой витамина D, обуславливающей все его эффекты [5-7].

\section{МЕХАНИЗМ ДЕЙСТВИЯ ВИТАМИНА D}

Механизм действия активной формы витамина D аналогичен механизму действия других стероидных гормонов и обусловлен его связыванием с ядерным рецептором [8]. 1,25(OH) 2 я явлется высокоаффинным лигандом для рецептора витамина D (VDR), который присутствует не только в кишечнике, костной ткани и почках - ключевых органах, ответственных за состояние фосфорно-кальциевого обмена, но и более чем в 38 различных органах-мишенях [9]. Их взаимодействие приводит к образованию гормон-рецепторного комплекса, изменяющего экспрессию генов путем связывания своего специфичного домена с регуляторной нуклеотидной последовательностью ДНК [10]. Таким образом происходит активация синтеза одних белков (например, кальций-связывающего белка, остеокальцина, остеопонтина) и угнетение образования других (провоспалительных цитокинов: ИЛ-6, 8) [11, 12].

В большом количестве наблюдательных работ показано наличие взаимосвязи низкого уровня витамина D c рядом различных системных заболеваний, например, сахарным диабетом, ИБС, застойной сердечной недостаточностью, артериальной гипертензией, аутоиммунными заболеваниями (такими как ревматоидный артрит, системная красная волчанка, рассеянный склероз, болезнь Крона), хронической болезнью почек и др. [13-17].

Регуляция витамином D фосфорно-кальциевого обмена и метаболизма костной ткани, а также оказание противовоспалительного и иммуномодулирующего эффектов, влияние на клеточную пролиферацию и дифференцировку могут существенно сказываться на состоянии полости рта $[6,18,19]$. В ряде работ продемонстрирована ассоциация низкого уровня витамина D с развитием и частотой рецидивов хронического афтозного стоматита, течением и ответом на лечение плоскоклеточной карциномы полости рта, тяжестью заболеваний пародонта, процессами остеоинтеграции и костного ремоделирования при дентальной имплантации и костно-пластических операциях [18, 20-26].

\section{СЛИЗИСТАЯ ОБОЛОЧКА РТА}

Хронический рецидивирующий афтозный стоматит (ХРАС) - хроническое заболевание слизистой оболочки полости рта, проявляющееся высыпаниями одиночных болезненных эрозий округлой или овальной формы с некрозом в центре и венчиком по периферии. Этиология данного заболевания до сих пор остается неизвестной, однако нарушение регуляции иммунного ответа относят к факторам риска наряду с генетическими дефектами, местной травмой, эмоциональным стрессом и дефицитом витаминов $[18,27,28]$.

Существенное участие витамина D в системе врожденного и приобретенного иммунитета, влияние на синтез провоспалительных цитокинов, обнаружение VDR на макрофагах, дендритных клетках, T- и В-лимфоцитах может объяснять его потенциальную связь с патогенезом ХРАC $[19,20,21]$.

Согласно ряду исследований $[18,20,21]$, у пациентов с ХРАС уровень сывороточного 25(OH)D был значительно ниже, чем у здоровых людей с аналогичными возрастными и гендерными параметрами. Таким образом, Aynure Öztekin and \& Coşkun Öztekin [18] рекомендуют прием витамина D в качестве поддерживающего лечения у пациентов с рецидивирующим афтозным стоматитом. Однако другое исследование, Ewa Krawiecka et al. [28], продемонстрировало отсутствие существенной разницы относительно уровня витамина D в сыворотке крови.

\section{ОНКОЛОГИЧЕСКИЕ ЗАБОЛЕВАНИЯ ПОЛОСТИ РТА}

Одним из наиболее распространенных злокачественных новообразований области головы и шеи является плоскоклеточная карцинома полости рта, которая ежегодно насчитывает более 300000 новых случаев во всем мире [29].

Под действием экзогенных и эндогенных факторов (курение табака, употребление алкоголя, воздействие вирусных инфекций, таких как вирус папилломы человека (ВПЧ), вирус Эпштейна-Барр, вирус гепатита С, ВИЧ) происходят молекулярные и клеточные изменения [30], которые проявляются в возникновении устойчивости к апоптозу раковых стволовых клеток, что продлевает их жизненный цикл $[22,31]$. Нарушение запрограммированной гибели клеток является ключевым фактором канцерогенеза плоскоклеточного рака полости рта 
и проявляется в выраженной устойчивости к радиои химиотерапии, большинству стандартных противоопухолевых препаратов [31, 32].

В связи с этим возник значительный интерес к изучению химиопрофилактического и терапевтического потенциала витамина D [33].

Противоопухолевая активность $1,25(\mathrm{OH})_{2} \mathrm{D}_{3}$ в ряде клеток обеспечивается его способностью индуцировать апоптоз, ингибировать инвазию, клеточную пролиферацию и ангиогенез опухоли [31, 32, 34].

B раковых клетках 1,25(OH), $\mathrm{D}_{3}$ активирует ингибиторы циклинзависимых киназ (р21, р27), митогенные факторы роста (IGF-1, EGF) и способствует активации TGF- $\beta$, таким образом проявляя антипролиферативные свойства [35].

Согласно исследованию [22], более чем у 74\% пациентов с плоскоклеточной карциномой полости рта обнаружено снижение уровня сывороточного витамина D по сравнению с контрольной группой, не имеющей рака в анамнезе. Положительная связь между риском возникновения плоскоклеточной карциномы и дефицитом витамина D, особенно при уровне ниже 25 нг/мл, повышает вероятность развития злокачественного новообразования в 1,65 раза [22].

Anand et al. пришли к заключению, что пациенты с плоскоклеточной карциномой полости рта, получавшие витамин $\mathrm{D}_{3}$ в дозе $1000 \mathrm{ME}$ в день на протяжении 3 мес, показали уменьшение побочных эффектов, связанных с химиотерапией. Отмечались снижение выраженности орального мукозита (уменьшение гиперемии, отека, изъязвлений и боли), улучшение функции глотания и повышение качества жизни по сравнению с пациентами, которые не получали витамин $D_{3}$ [32].

\section{ОСТЕОИНТЕГРАЦИЯ}

К наиболее изученным и доказанным эффектам витамина D относят поддержание фосфорно-кальциевого гомеостаза и регуляцию костного ремоделирования, которые реализуются через повышение абсорбции кальция в кишечнике, усиление реабсорбции в почечных канальцах, подавление синтеза и секреции паратиреоидного гормона, активацию остеокластов, увеличение продукции внеклеточного матрикса остеобластами и экспрессии генов остеокальцина, остеопонтина, кальбиндина и 24-гидроксилазы [36-40]. Исходя из этих данных, витамин D может играть существенную роль в регенерации костной ткани при дентальной имплантации и костно-пластических операциях.

Достижение долгосрочного функционирования дентальных имплантатов обеспечивается их остеоинтеграцией, которая характеризуется прямой прочной связью между костью и поверхностью имплантата при отсутствии фиброзной ткани. Данное явление зависит от многих факторов: материала, конструкции и поверхности имплантата, хирургической техники и ортопедического протокола лечения, а также качества кости и регенеративных возможностей организма $[41,42]$.

Процесс остеоинтеграции дентальных имплантатов, состоящий из нескольких этапов, сопровождается активной остеокластической резорбцией недифференцированного костного вещества $[43,44]$. Формирование кости после костнопластических операций происходит через резорбцию трансплантата и замену трансплантата собственной костной тканью пациента [23]. В этих метаболических процессах остеокласты выполняют активную роль наравне с остеобластами [43].

Поскольку остеоинтеграция дентальных имплантатов зависит от способности кости к регенерации, предполагается, что образование костной ткани вокруг имплантата снижается при дефиците витамина D [39, 41, 45].

Fretwurst et al. [36] предоставили два клинических случая раннего отторжения имплантата у пациентов с дефицитом витамина D. B этой статье показано, что последующая установка имплантатов была успешной после приема витамина D и корректировки его уровня в сыворотке крови.

Клинический случай, продемонстрированный Bryce G., MacBeth N. [24], говорит об отсутствии интеграции спустя 5 мес после одномоментной имплантации у пациента с выраженным дефицитом витамина D, который мог способствовать отторжению дентального имплантата.

В своем исследовании Schulze-Späte et al. [23] отмечают значительную связь между повышенным уровнем сывороточного витамина D и присутствием остеокластов вокруг частиц трансплантата при аугментации, что может указывать на более выраженную метаболическую активность, которая способствует локальному ремоделированию в зоне аугментации.

Однако систематический обзор Javed et al. [39] показал, что влияние уровня витамина D на остеоинтеграцию имплантатов до сих пор остается спорным.

Mangano et al. [38, 46] в своих ретроспективных исследованиях смогли проследить тенденцию к увеличению частоты ранних отторжений имплантатов у пациентов с тяжелым дефицитом витамина D, однако доказать достоверность связи между этими фактами так и не получилось, так как статистически значимая разница не была выявлена.

Согласно исследованиям $[46,47]$, предоперационный контроль уровня 25(OH)D в сыворотке крови и при необходимости назначение врачом витамина D за несколько недель до операции могут ускорить процесс заживления и предотвратить возникновение раннего отторжения имплантата.

Активная форма витамина D - 1,25(OH) 2 D может влиять на остеоинтеграцию и через процессы, связанные с заживлением и краевым прилеганием мягких тканей, окружающих имплантат, и способствовать снижению развития бактериальных инфекций и периимплантитов [48].

В работе Acipinar S. et al. [49] было зафиксировано значительное снижение 25(OH)D в группе с периимплантитом в сравнении с контрольной здоровой группой.

\section{ЗАБОЛЕВАНИЯ ПАРОДОНТА}

В современной практике врача-стоматолога достаточно остро стоит проблема хронического генерализованного пародонтита. Это связано не только с высокой распространенностью этой патологии среди населения, но и с отсутствием ожидаемого эффекта от лечения как для пациента, так для врача [50].

Достаточно часто врачи-стоматологи сталкиваются с резистентностью лечения хронического генерализованного пародонтита, уменьшением сроков стойкой ремиссии и учащением агрессивного течения пародонтита. Представленные проблемы демонстрируют 
необходимость более тщательного изучения компонентов патогенеза хронического генерализованного пародонтита и поиска решений комплексного лечения на основании полученных данных [50].

Пародонтит характеризуется поражением тканей, окружающих зуб, вызванным иммуновоспалительным ответом организма на бактериальную инвазию. Поскольку витамин D играет решающую роль в метаболизме костной ткани и поддержании иммунного ответа, есть основание предполагать, что его дефицит может влиять на патогенез заболевания и негативно сказываться на состоянии пародонта [51, 52].

Активный метаболит витамина $\mathrm{D}-1,25(\mathrm{OH})_{2} \mathrm{D}_{3}$ участвует в специфической иммунной защите и оказывает противовоспалительный эффект, воздействуя на Т- и В-лимфоциты, ингибируя выработку провоспалительных ИЛ-6 и ИЛ-8, которые участвуют в развитии острого воспаления [25, 52-55].

Регуляция неспецифического иммунного ответа происходит путем стимуляции синтеза антимикробных пептидов (дефензинов и кателицидина) через рецепторы витамина D (VDR), находящиеся в моноцитах, макрофагах, нейтрофилах и дендритных клетках $[3,4]$.

Один из дефензинов - бета-дефензин 2 проявляет антимикробную активность против патогенов полости рта, включая бактерии, ассоциированные с развитием пародонтита (Porphyromonas gingivalis, Fusobacterium nucleatum и Aggregatibacter actinomycetemcomitans) $[25,56]$.

Анализ данных, представленных Bashutski J.D. et al. [51], показывает, что дефицит витамина D приводит к менее эффективным результатам (более низкий уровень прикрепления мягких тканей и изменение глубины зондирования) после операций, производимых на пародонте.

Pinto et al. [57] в своем систематическом обзоре утверждают, что связь между заболеваниями пародонта и дефицитом витамина D может быть вполне обоснована, однако большинство исследований имеют значительные ограничения, что не позволяет подтвердить наличие данной связи.

Исследования Isola et al. [26], а также Anbarcioglu E. et al. [25] показали, что пациенты с пародонтитом имели более низкие уровни витамина D в сыворотке по сравнению со здоровыми пациентами. Более того, дефицит витамина D отрицательно влиял на течение заболеваний пародонта и повышал риск возникновения агрессивного пародонтита. Поскольку это исследование подтверждает связь между низким уровнем витамина D в сыворотке крови и развитием пародонтита, по мнению авторов, оценка уровня витамина D должна быть рекомендована в начале пародонтальной терапии, так как это может уменьшить риск развития данного заболевания [26].

В добавление к вышесказанному, исследование Garcia et al. демонстрирует, что препараты кальция и витамина D (1000 ME/день) оказывали умеренное положительное влияние на состояние пародонта и улучшили клинические параметры. Данные результаты подтверждают возможность положительного влияния витамина D на здоровье пародонта [58].

\section{ЗАКЛЮЧЕНИЕ}

Витамин D, участвуя в фосфорно-кальциевом метаболизме костной ткани челюстно-лицевой области, оказывает активное влияние на процессы остеоинтеграции и ремоделирования кости. Данный эффект может способствовать снижению риска раннего отторжения дентальных имплантатов, благоприятному исходу костно-пластических операций при аугментациях, а также снижению деструктивных изменений при тяжелом течении пародонтита. Обнаружение «внекостных» эффектов витамина D позволило шире взглянуть на его значимость при заболеваниях челюстно-лицевой области. Участие витамина D в иммунных процессах, оказание противовоспалительного и антимикробного эффектов, замедление клеточной пролиферации и стимуляция дифференцировки обуславливают развитие и течение ХРАС, плоскоклеточной карциномы полости рта, пародонтита и заживление мягких тканей после имплантации и пародонтологических операций.

На сегодняшний день имеются отдельные работы, свидетельствующие о наличии ассоциации между низким уровнем витамина $\mathrm{D}$ и заболеваниями челюстно-лицевой области. Для уточнения причинно-следственной связи, а также для решения вопроса о целесообразности оценки статуса витамина D и необходимости компенсации его дефицита целесообразно проведение дальнейших исследований.

\section{ДОПОЛНИТЕЛЬНАЯ ИНФОРМАЦИЯ}

Источники финансирования. Работа выполнена по инициативе авторов без привлечения финансирования.

Конфликт интересов. Авторы декларируют отсутствие явных и потенциальных конфликтов интересов, связанных с содержанием настоящей статьи.

Участие авторов. Все авторы одобрили финальную версию статьи перед публикацией, выразили согласие нести ответственность за все аспекты работы, подразумевающую надлежащее изучение и решение вопросов, связанных с точностью или добросовестностью любой части работы.

\section{СПИСОК ЛИТЕРАТУРЫ | REFERENCES}

1. Palacios C, Gonzalez L. Is vitamin D deficiency a major global public health problem? J Steroid Biochem Mol Biol. 2014;144:138-145. doi: https://doi.org/10.1016/j.jsbmb.2013.11.003

2. Пигарова Е.А., Рожинская Л.Я., Белая Ж.Е., и др. Клинические рекомендации Российской ассоциации эндокринологов по диагностике, лечению и профилактике дефицита витамина D у взрослых // Проблемы эндокринологии. — 2016. - Т. 62. №4. - C. 60-84. [Pigarova EA, Rozhinskaya LY, Belaya JE, et al. Russian Association of Endocrinologists recommendations for diagnosis, treatment and prevention of vitamin D deficiency in adults. Problems of Endocrinology. 2016;62(4):60-84. (In Russ.)]. doi: https://doi.org/10.14341/probl201662460-84

3. McMahon L, Schwartz K, Yilmaz O, et al. Vitamin D-mediated induction of innate immunity in gingival epithelial cells. Infection and immunity. 2011;79(6):2250-2256. doi: https://doi.org/10.1128/IAl.00099-11

4. Krawiec M, Dominiak M. The role of vitamin D in the human body with a special emphasis on dental issues: Literature review. Dent Med Probl. 2018;55(4):419-424. doi: https://doi.org/10.17219/dmp/99051 
5. Мальцев С.В., Мансурова Г.Ш. Метаболизм витамина D и пути реализации его основных функций // Практическая медицина. 2014. - T. 9. - №85. - C. 12-18. [Mal'tsev SV, Mansurova GSh. Metabolizm vitamina D i puti realizatsii ego osnovnykh funktsii. Prakticheskaya meditsina. 2014;9(85):12-18. (In Russ.)].

6. Ślebioda Z, Szponar E, Dorocka-Bobkowska B. Vitamin D and Its Relevance in the Etiopathogenesis of Oral Cavity Diseases. Arch Immunol Ther Exp (Warsz). 2016;64(5):385-397. doi: https://doi.org/10.1007/s00005-016-0384-z

7. Grygiel-Górniak B, Puszczewicz M. Vitamin D - a new look in medicine and rheumatology. Postepy Hig Med Dosw. 2014;68(5):359-368. doi: https://doi.org/10.5604/17322693.1097426

8. Bikle D. Nonclassic actions of vitamin D. The Journal of Clinical Endocrinology \& Metabolism. 2009;94(1):26-34. doi: https://doi.org/10.1210/jc.2008-1454

9. Norman AW, Bouillon R. Vitamin D nutritional policy needs a vision for the future. Experimental Biology and Medicine. 2010;235(9):1034-1045. doi: https://doi.org/10.1258/ebm.2010.010014

10. Adams JS, Hewison M. Update in vitamin D. The Journal of Clinical Endocrinology \& Metabolism. 2010;95(2):471-478. doi: https://doi.org/10.1210/jc.2009-1773

11. Tang $X$, Pan $Y, Z$ Zhao Y. Vitamin D inhibits the expression of interleukin-8 in human periodontal ligament cells stimulated with Porphyromonas gingivalis. Archives of Oral Biology. 2013;58(4):397-407. doi: https://doi.org/10.1016/j.archoralbio.2012.09.010

12. Nebel D, Svensson D, Arosenius $K$, et al. 1a,25-dihydroxyvitamin D3 promotes osteogenic activity and downregulates proinflammatory cytokine expression in human periodontal ligament cells. Journal of Periodontal Research. 2014;50(5):666-673. doi: https://doi.org/10.1111/jre.12249

13. Lavie CJ, Lee JH, Milani RV. Vitamin D and cardiovascular disease: will it live up to its hype? Journal of the American College of Cardiology. 2011;58(15):1547-1556 doi: https://doi.org/10.1016/j.jacc.2011.07.008

14. Goel RK, Lal H. Role of vitamin d supplementation in hypertension. Indian Journal of Clinical Biochemistry. 2011;26(1):88-90. doi: https://doi.org/10.1007/s12291-010-0092-0

15. Agmon-Levin N, Blank M, Zandman-Goddard G, et al. Vitamin D: An instrumental factor in the anti-phospholipid syndrome by inhibition of tissue factor expression. Ann Rheum Dis. 2011. doi: https://doi.org/10.1136/ard.2010.134817

16. Wang $X X$, Jiang $T$, Shen $Y$, et al. Vitamin $D$ receptor agonist doxercalciferol modulates dietary fat-induced renal disease and renal lipid metabolism. Am J Physiol Physiol. 2011;300(3):F801-F810. doi: https://doi.org/10.1152/ajprenal.00338.2010

17. Niroomand M, Fotouhi A, Irannejad N, Hosseinpanah F. Does high-dose vitamin D supplementation impact insulin resistance and risk of development of diabetes in patients with pre-diabetes? A double-blind randomized clinical trial. Diabetes Res Clin Pract. 2019;148:1-9. doi: https://doi.org/10.1016/j.diabres.2018.12.008

18. Öztekin A, Öztekin C. Vitamin D levels in patients with recurrent aphthous stomatitis. BMC Oral Health. 2018;18(1):1-5. doi: https://doi.org/10.1186/s12903-018-0653-9

19. Khammissa RAG, Fourie J, Motswaledi MH, Ballyram R, Lemmer J Feller L. The Biological Activities of Vitamin D and Its Receptor in Relation to Calcium and Bone Homeostasis, Cancer, Immune and Cardiovascular Systems, Skin Biology, and Oral Health. Biomed Res Int. 2018;2018:1-9. doi: https://doi.org/10.1155/2018/9276380

20. Bahramian A, Falsafi, P, Abbasi T, et al. Comparing serum and salivary levels of vitamin D in patients with recurrent aphthous stomatitis and healthy individuals. Journal of Dentistry. 2018;19(4):295.

21. Khabbazi A, Ghorbanihaghjo A, Fanood F, et al. A comparative study of vitamin D serum levels in patients with recurrent aphthous stomatitis. Egypt Rheumatol. 2015;37(3):133-137. doi: https://doi.org/10.1016/j.ejr.2014.07.005

22. Udeabor SE, Albejadi AM, Al-Shehri WAK, et al. Serum levels of 25-hydroxy-vitamin $D$ in patients with oral squamous cell carcinoma: Making a case for chemoprevention. Clin Exp Dent Res. 2020;6(4):428-432. doi: https://doi.org/10.1002/cre2.294

23. Schulze-Späte U, Dietrich T, Wu C, Wang K, Hasturk H, Dibart S. Systemic vitamin D supplementation and local bone formation after maxillary sinus augmentation — a randomized, double-blind, placebo-controlled clinical investigation. Clin Oral Implants Res. 2016;27(6):701-706. doi: https://doi.org/10.1111/clr.12641
24. Bryce $\mathrm{G}$, MacBeth N. Vitamin D deficiency as a suspected causative factor in the failure of an immediately placed dental implant: a case report. J R Nav Med Serv. 2014. doi: https://doi.org/10.1136/jrnms-100-328

25. Anbarcioglu E, Kirtiloglu T, Öztürk A, et al. Vitamin D deficiency in patients with aggressive periodontitis. Oral diseases. 2019;25(1):242-249. doi: https://doi.org/ 10.1111/odi.12968

26. Isola G, Alibrandi A, Rapisarda E, et al. Association of vitamin D in patients with periodontitis: A cross-sectional study. Journal of Periodontal Research. 2020; 55(5):602-612 doi: https://doi.org/ 10.1111/jre.12746

27. Al-Maweri SA, Halboub E Al-Sufyani G, et al. Is vitamin D deficiency a risk factor for recurrent aphthous stomatitis? A systematic review and meta-analysis. Oral Diseases. 2020; 26(6):1116-1123. doi: https://doi.org/10.1111/odi.13189

28. Krawiecka E, Ślebioda Z, Szponar E, et al. Vitamin D status in recurrent aphthous stomatitis. Advances in Dermatology and Allergology/Postępy Dermatologii i Alergologii. 2017; 34(6):612. doi: https://doi.org/10.5114/pdia.2017.69683

29. Rivera C, Oliveira AK, Costa RAP, et al. Prognostic biomarkers in oral squamous cell carcinoma: A systematic review. Oral Oncology. 2017;72:38-47. doi: https://doi.org/10.1016/j.oraloncology.2017.07.003

30. Markopoulos AK. Current Aspects on Oral Squamous Cell Carcinoma. Open Dent J. 2012;6(1):126-130. doi: https://doi.org/10.2174/1874210601206010126

31. Grimm M, Cetindis M, Biegner T, et al. Serum vitamin D levels of patients with oral squamous cell carcinoma (OSCC) and expression of vitamin D receptor in oral precancerous lesions and OSCC. Med Oral Patol Oral y Cir Bucal. 2015;20(2):e188-e195. doi: https://doi.org/10.4317/medoral.20368

32. Anand A, Singh S, Sonkar AA, et al. Expression of vitamin D receptor and vitamin D status in patients with oral neoplasms and effect of vitamin D supplementation on quality of life in advanced cancer treatment. Współczesna Onkol. 2017;2:145-151. doi: https://doi.org/10.5114/wo.2017.68623

33. Verma A, Vincent-Chong VK, DeJong $\mathrm{H}$, et al. Impact of dietary vitamin D on initiation and progression of oral cancer. J Steroid Biochem Mol Biol. 2020;199:105603. doi: https://doi.org/10.1016/j.jsbmb.2020.105603

34. Osafi J, Hejazi A, Stutz DD, Keiserman MA, et al. Differentia Effects of 1,25-dihydroxyvitamin D 3 on Oral Squamous Cell Carcinomas In Vitro. J Diet Suppl. 2014;11 (2):145-154 doi: https://doi.org/10.3109/19390211.2013.859209

35. Christakos S, Dhawan P, Verstuyf A, et al. Vitamin D: metabolism, molecular mechanism of action, and pleiotropic effects. Physiological reviews. 2016;96(1):365-408. doi: https://doi.org/10.1152/physrev.00014.2015

36. Fretwurst T, Grunert S, Woelber JP, et al. Vitamin D deficiency in early implant failure: two case reports. Int J Implant Dent. 2016;2(1):24. doi: https://doi.org/10.1186/s40729-016-0056-0

37. 1Choukroun J, Khoury G, Khoury F, et al. Two Neglected Biologic Risk Factors in Bone Grafting and Implantology: High Low-Density Lipoprotein Cholesterol and Low Serum Vitamin D. J Oral Implantol. 2014;40(1):110-114. doi: https://doi.org/10.1563/AAID-JOI-D-13-00062

38. Mangano F, Mortellaro C, Mangano N, Mangano C. Is Low Serum Vitamin D Associated with Early Dental Implant Failure? A Retrospective Evaluation on 1625 Implants Placed in 822 Patients. Mediators Inflamm. 2016;2016:1-7. doi: https://doi.org/10.1155/2016/5319718

39. Javed F, Malmstrom H, Kellesarian SV, et al. Efficacy of Vitamin D3 Supplementation on Osseointegration of Implants. Implant Dent. 2016;25(2):281-287. doi: https://doi.org/10.1097/ID.0000000000000390

40. Haussler MR, Whitfield GK, Kaneko I, et al. Molecular Mechanisms of Vitamin D Action. Calcif Tissue Int. 2013;92(2):77-98. doi: https://doi.org/10.1007/s00223-012-9619-0

41. Trindade R, Albrektsson T, Wennerberg A. Current Concepts for the Biological Basis of Dental Implants. Oral Maxillofac Surg Clin North Am. 2015;27(2):175-183. doi: https://doi.org/10.1016/j.coms.2015.01.004

42. Troiano G, Lo Russo L, Canullo L, et al. Early and late implant failure of submerged versus non-submerged implant healing: A systematic review, meta-analysis and trial sequential analysis. J Clin Periodontol. 2018;45(5):613-623. doi: https://doi.org/10.1111/jcpe.12890 
43. Insua A, Monje A, Wang H-L, Miron RJ. Basis of bone metabolism around dental implants during osseointegration and peri-implant bone loss. J Biomed Mater Res Part A. 2017;105(7):2075-2089. doi: https://doi.org/10.1002/jbm.a.36060

44. Мирсаева Ф.З., Убайдуллаев М.Б., Вяткина А.Б., Фаткуллина С.Ш. Дентальная имплантология / Под ред. Ф.З. Мирсаевой. - Уфа: Изд-во ГБОУ ВПО БГМУ Минздрава России; 2015. - 124 с. [Mirsaeva FZ, Ubaidullaev MB, Vyatkina AB, Fatkullina SSh. Dental'naya implantologiya. Ed. by FZ. Mirsaeva. Ufa: Izd-vo GBOU VPO BGMU Minzdrava Rossii; 2015. 124 p. (In Russ.)]

45. Apostu D, Lucaciu O, Lucaciu GDO, et al.

Systemic drugs that influence titanium implant osseointegration. Drug Metab Rev. 2017;49(1):92-104 doi: https://doi.org/10.1080/03602532.2016.1277737

46. Guido Mangano F, Ghertasi Oskouei S, Paz A, et al. Low serum vitamin $D$ and early dental implant failure: Is there a connection? A retrospective clinical study on 1740 implants placed in 885 patients. J Dent Res Dent Clin Dent Prospects. 2018;12(3):174-182. doi: https://doi.org/10.15171/joddd.2018.027

47. Waskiewicz K, Oth O, Kochan N, Evrard L. Risk factors generally neglected in oral surgery and implantology: the high LDL-cholesterol and the insufficient level of vitamin D. Rev Med Brux. 2018:39(2):70-77. doi: https://doi.org/10.30637/2018.17-075

48. Nastri L, Moretti A, Migliaccio S, et al. Do Dietary Supplements and Nutraceuticals Have Effects on Dental Implant Osseointegration? A Scoping Review. Nutrients. 2020;12(1):268. doi: https://doi.org/10.3390/nu12010268

49. Acipinar S, Karsiyaka Hendek M, Olgun E, Kisa U. Evaluation of FGF-23 and 25(OH)D 3 levels in peri-implant sulcus fluid in peri-implant health and diseases. Clin Implant Dent Relat Res. 2019;21(5):1106-1112. doi: https://doi.org/10.1111/cid.12832

50. Aimetti M, Perotto S, Castiglione A, et al. Prevalence of periodontitis in an adult population from an urban area in North Italy: findings from a cross-sectional population-based epidemiological survey. J Clin Periodontol. 2015:42(7):622-631. doi: https://doi.org/10.1111/jcpe.12420

51. Bashutski JD, Eber RM, Kinney JS, et al. The Impact of Vitamin D Status on Periodontal Surgery Outcomes. J Dent Res. 2011;90(8):1007-1012. doi: https://doi.org/10.1177/0022034511407771

52. Laky M, Bertl K, Haririan H, et al. Serum levels of 25-hydroxyvitamin D are associated with periodontal disease. Clin Oral Investig. 2017;21(5):1553-1558. doi: https://doi.org/10.1007/s00784-016-1965-2

53. Jagelavičienè E, Vaitkevičienè I, Šilingaitè D, et al. The Relationship between Vitamin D and Periodontal Pathology. Medicina (B Aires). 2018;54(3):45. doi: https://doi.org/10.3390/medicina54030045

54. Agrawal AA, Kolte AP, Kolte RA, et al. Evaluation and comparison of serum vitamin D and calcium levels in periodontally healthy, chronic gingivitis and chronic periodontitis in patients with and without diabetes mellitus a cross-sectional study. Acta Odontol Scand. 2019;77(8):592-599. doi: https://doi.org/10.1080/00016357.2019.1623910

55. Elenkova M, Tipton DA, Karydis A, Stein SH. Vitamin D attenuates human gingival fibroblast inflammatory cytokine production following advanced glycation end product interaction with receptors for AGE. J Periodontal Res. 2019;54(2):154-163. doi: https://doi.org/10.1111/jre.12613

56. Dragonas P, El-Sioufi I, Bobetsis YA, Madianos PN Association of Vitamin D with periodontal disease: A narrative review. Oral Heal Prev Dent. 2020;18:103-114. doi: https://doi.org/10.3290/j.ohpd.a44323

57. Pinto JPNS, Goergen J, Muniz FWMG, Haas AN. Vitamin D levels and risk for periodontal disease: A systematic review. J Periodontal Res. 2018;53(3):298-305. doi: https://doi.org/10.1111/jre.12531

58. Garcia MN, Hildebolt CF, Miley DD, et al. One-Year Effects of Vitamin D and Calcium Supplementation on Chronic Periodontitis. J Periodontol. 2011;82(1):25-32. doi: https://doi.org/10.1902/jop.2010.100207

\section{ИНФОРМАЦИЯ ОБ АВТОРАХ [AUTHORS INFO]}

* Дьячкова Екатерина Юрьевна, к.м.н. [Ekaterina Y. Diachkova, PhD]; Россия, 119991, Москва, ул. Трубецкая, д. 8, стр. 2 [8-2 Trubetskaya str., Moscow, Russian Federation, 119991]; ORCID: https://orcid.org/0000-0003-4388-8911; eLibrary SPIN: 6877-3782; e-mail: secu2003@mail.ru

Трифонова Дарья Олеговна, студент [Daria O. Trifonova, student]; ORCID: https://orcid.org/0000-0003-2414-6437; e-mail: trifonova030897@mail.ru

Ибадулаева Мария Олеговна, ординатор [Maria O. Ibadulaeva, resident]; ORCID: https://orcid.org/0000-0002-3961-091X; e-mail: tmo-4509@mail.ru

Рунова Гюзель Евгеньевна, д.м.н. [Guzel E. Runova, MD]; ORCID: https://orcid.org/0000-0003-2144-8595;

eLibrary SPIN: 3639-6932; e-mail: guzelvolkova@yandex.ru

Фадеев Валентин Викторович, д.м.Н., профессор, член-корр. PAH [Valentin V. Fadeyev, MD, PhD, Professor, correspondence fellow of Russian Academy of Sciences]; ORCID: https://orcid.org/0000-0002-3026-6315;

eLibrary SPIN: 6825-8417; e-mail: walfad@mail.ru

Тарасенко Светлана Викторовна, д.м.Н., профессор [Svetlana V. Tarasenko, MD, Professor];

ORCID: http://orcid.org/0000-0001-8595-8864; eLibrary SPIN: 3320-0052; e-mail: prof_tarasenko@rambler.ru

\section{ИНФОРМАЦИЯ}

Рукопись получена: 26.03.2021. Одобрена к публикации: 26.05.2021.

\section{ЦИТИРОВАТЬ:}

Дьячкова Е.Ю., Трифонова Д.О., Ибадулаева М.О., Рунова Г.Е., Фадеев В.В., Тарасенко С.В. Влияние дефицита витамина D на состояние зубочелюстной системы: обзорная статья // Остеопороз и остеопатии. — 2021. — Т. 24. — №1. — C. 19-25. doi: https://doi.org/10.14341/osteo12719

\section{TO CITE THIS ARTICLE:}

Diachkova EY, Trifonova DO, Ibadulaeva MO, Runova GE, Fadeev VV, Tarasenko SV. Vitamin D imbalance effects on dentoalveolar system: a review. Osteoporosis and bone diseases. 2021;24(1):19-25. doi: https://doi.org/10.14341/osteo12719 